Gut and Liver, Vol. 13 No. 4, July 2019, pp. 402-408

\title{
A Pilot Study of Endoscopic Submucosal Dissection Using an Endoscopic Assistive Robot in a Porcine Stomach Model
}

\author{
Byung Gon Kim ${ }^{1}$, Hyuk Soon Choi ${ }^{2}$, Sei Hoon Park ${ }^{1}$, Jun Ho Hong ${ }^{1}$, Jung Min Lee², Seung Han Kim² ${ }^{2}$ Hoon Jai Chun², \\ Daehie Hong ${ }^{1}$, and Bora Keum ${ }^{2}$ \\ ${ }^{1}$ Department of Mechanical Engineering, Korea University, and ${ }^{2}$ Division of Gastroenterology and Hepatology, Department of Internal \\ Medicine, Institute of Gastrointestinal Medical Instrument Research, Korea University College of Medicine, Seoul, Korea
}

\section{See editorial on page 381}

Background/Aims: Endoscopic assistive devices have been developed to reduce the complexity and improve the safety of surgeries involving the use of endoscopes. We developed an assistive robotic arm for endoscopic submucosal dissection (ESD) and evaluated its efficiency and safety in this in vitro pilot study. Methods: ESD was performed using an auxiliary transluminal endoscopic robot. An in vitro test bed replicating the intra-abdominal environment and pig stomachs were used for the experiment. Participants were divided into skilled operators and unskilled operators. Each group performed ESD 10 times by using both conventional and robot-assisted methods. The perforation incidence, operation time, and resected mucous membrane size were measured. Results: For the conventional method, significant differences were noted between skilled and unskilled operators regarding operation time (11.3 minutes vs 26.7 minutes) and perforation incidence (0/10 vs 6/10). Unskilled operators showed a large decrease in the perforation incidence with the robot-assisted method (conventional method vs robot-assisted method, 6/10 vs 1/10). However, the operation time did not differ between the conventional and robotassisted methods. On the other hand, skilled operators did not show differences in the operation time and perforation incidence between the conventional and robot-assisted methods. Among both skilled and unskilled operators, the operation time decreased with the robot-assisted method as the experiment proceeded. Conclusions: The surgical safety of unskilled operators greatly improved with robotic assistance. Thus, our assistive robotic arm was beneficial for ESD. Our findings suggest that endoscopic assistive robots have positive effects on surgical safety. (Gut Liver 2019;13:402408)

Key Words: Endoscopic mucosal resection; Endoscopy; Pilot projects; Surgery, computer-assisted

\section{INTRODUCTION}

Currently, studies on minimally invasive surgery involving endoscopes are being actively performed worldwide. ${ }^{1-4}$ Among various surgeries involving the use of endoscopes, endoscopic submucosal dissection (ESD) is an effective surgical method for early gastrointestinal neoplasms., ${ }^{5,6}$ However, ESD requires experienced and highly skilled doctors because of the elaborate procedure and relatively limited degrees of freedom (DOFs) of endoscopes for performing required complex movements. ${ }^{7,8}$ Globally, various assistive devices have been developed to reduce the difficulty of highly complex endoscopic surgeries, such as ESD, and to employ endoscopes as useful surgical equipment. $^{9-14}$ These devices include EndoSamurai (Olympus Medical Systems Corp., Tokyo, Japan), EndoMaster (Nanyang Technological University, Singapore), Anubis (Karl Storz, Tuttlingen, Germany), and ViaCath (Hansen Medical, Mountain View, CA, USA). ${ }^{15-21}$ Moreover, studies on surgeries performed by unskilled operators using assistive robots are being conducted. ${ }^{22}$

Correspondence to: Daehie Hong ${ }^{\mathrm{a}}$ and Bora Keum ${ }^{\mathrm{b}}$

${ }^{a}$ Department of Mechanical Engineering, Korea University, 145 Anam-ro, Seongbuk-gu, Seoul 02841, Korea

Tel: +82-2-3290-3369, Fax: +82-2-926-9290, E-mail: dhhong@korea.ac.kr

${ }^{b}$ Division of Gastroenterology and Hepatology, Department of Internal Medicine, Korea University College of Medicine, 73 Inchon-ro, Seongbuk-gu, Seoul 02841, Korea

Tel: +82-2-920-6555, Fax: +82-2-953-1943, E-mail: borakeum@korea.ac.kr

Received on August 21, 2018. Revised on October 1, 2018. Accepted on October 15, 2018.

pISSN 1976-2283 eISSN 2005-1212 https://doi.org/10.5009/gnl18370

Byung Gon Kim and Hyuk Soon Choi contributed equally to this work as first authors.

(a) This is an Open Access article distributed under the terms of the Creative Commons Attribution Non-Commercial License (http://creativecommons.org/licenses/by-nc/4.0) which permits unrestricted non-commercial use, distribution, and reproduction in any medium, provided the original work is properly cited. 
Our research team developed a revolute joint-based auxiliary transluminal endoscopic robot (REXTER) for ESD. As an endoscopic assistive robot, REXTER can be attached or detached from an existing general-use endoscope. REXTER also enables complex movements by providing an additional range of motion. In the present study, we assessed the characteristics of REXTER and conducted an in vitro pilot test to evaluate the efficiency of REXTER.

\section{MATERIALS AND METHODS}

The present study was approved by the Laboratory Animal Research Center of Korea University College of Medicine (IRB number: KOREA 2017-0056).

\section{Endoscopic submucosal dissection}

We conducted a comparative study by using a conventional method and a robot-assisted method to verify the feasibility and safety of robot-assisted ESD. First, marking dots were made in the area around a lesion (diameter, 3 to $5 \mathrm{~cm}$ ) by using an electrosurgical dual knife (Olympus), and the mucous layer was lifted from the muscular layer by using saline solution and a 23-gauge injection needle. Next, circumferential precutting of the marked area was performed using the dual knife along the marking dots (Fig. 1A-C). An electrosurgical unit (ERBE VIO300D; Erbe Elektromedizin, Tübingen, Germany) was used during all procedures. These procedures were identically performed for both ESD methods. Following the procedures, only the endo- scope and dual knife were used to dissect the submucosal layer in the conventional method, whereas the assistive robot was used in the robot-assisted method (Fig. 1D-G). After surgery, the dissected mucous tissue was collected (Fig. 1H). The perforation incidence in the serosal layer during surgery was considered a standard to determine surgical safety. The operation time and dissected tissue area were recorded to evaluate the efficiency of the surgery.

\section{Revolute joint-based auxiliary transluminal endoscopic robot}

In this study, REXTER, an endoscopic, detachable, assistive robot, was developed to increase the efficiency and safety of ESD. During dissection of the submucosal layer of a lesion, the membrane dissected in the initial stage of surgery hides the area that must be subsequently dissected, thereby making it difficult to dissect the submucosal layer as the surgery proceeds (Fig. 1C). This factor can complicate ESD. Although skilled operators can complete the surgery with gravity, cap and superior dexterity after gaining adequate clinical experience, unskilled operators find it challenging to complete the surgery when the area intended for dissection cannot be identified. During ESD, REXTER provides operators with increased visibility of the dissection area as it lifts the mucosal flap dissected earlier. Therefore, operators can perform ESD while accurately identifying the dissection area until the end of the surgery.

REXTER includes a robot arm, actuator housing, and interface console (Fig. 2A). The robot arm can be installed at the
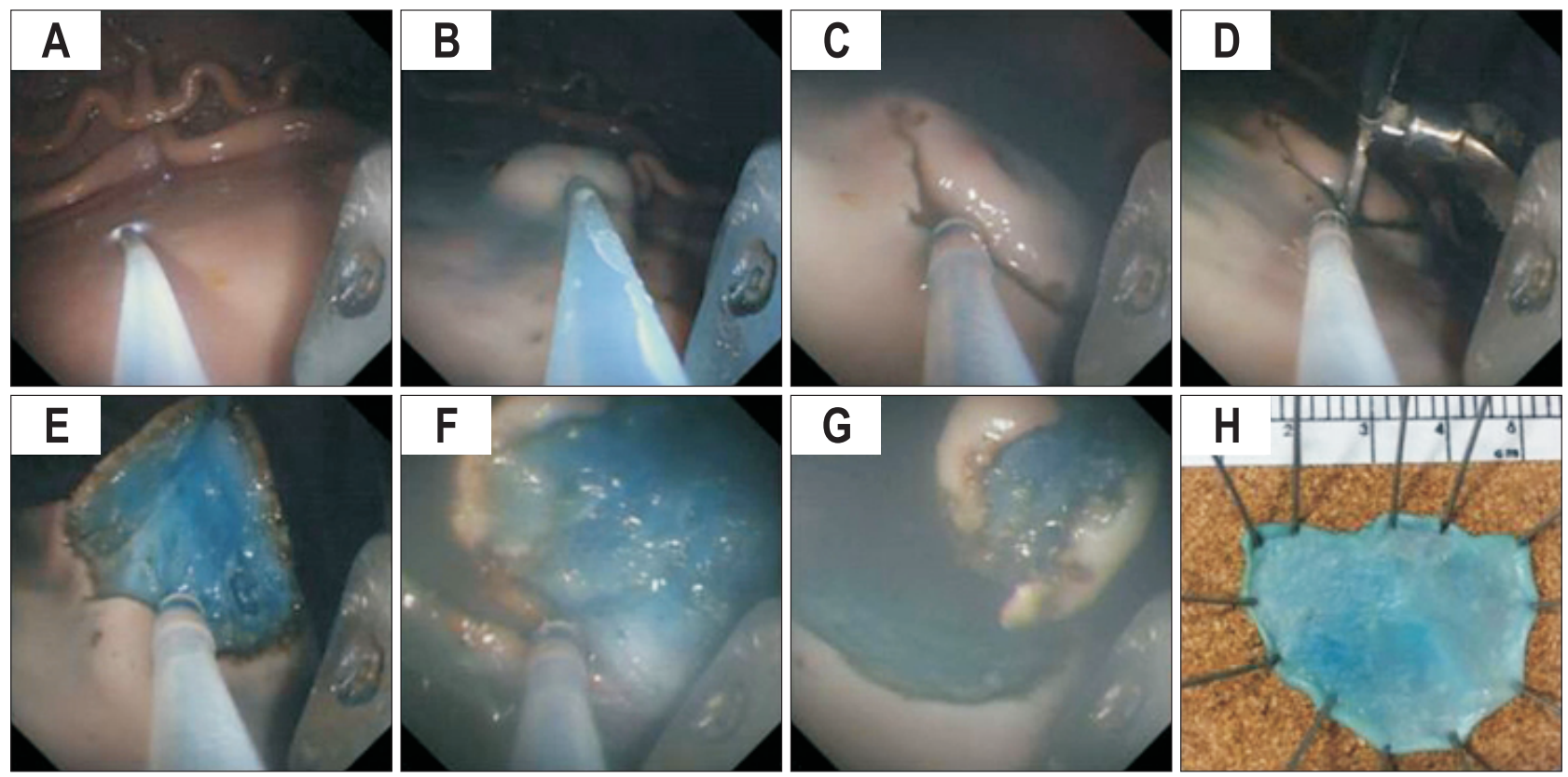

Fig. 1. Endoscopic submucosal dissection with an auxiliary endoscopic robot. (A) Making marking dots using a dual knife. (B) Injecting saline solution to the submucosal layer. (C) Circumferential precutting was performed by the dual knife. (D, E) Grasping and lifting up the mucous tissue using the assistive robot. (F, G) Dissecting submucous layer using the dual knife and the assistive robot. (H) Measuring the diameter of dissected mucous tissue. 

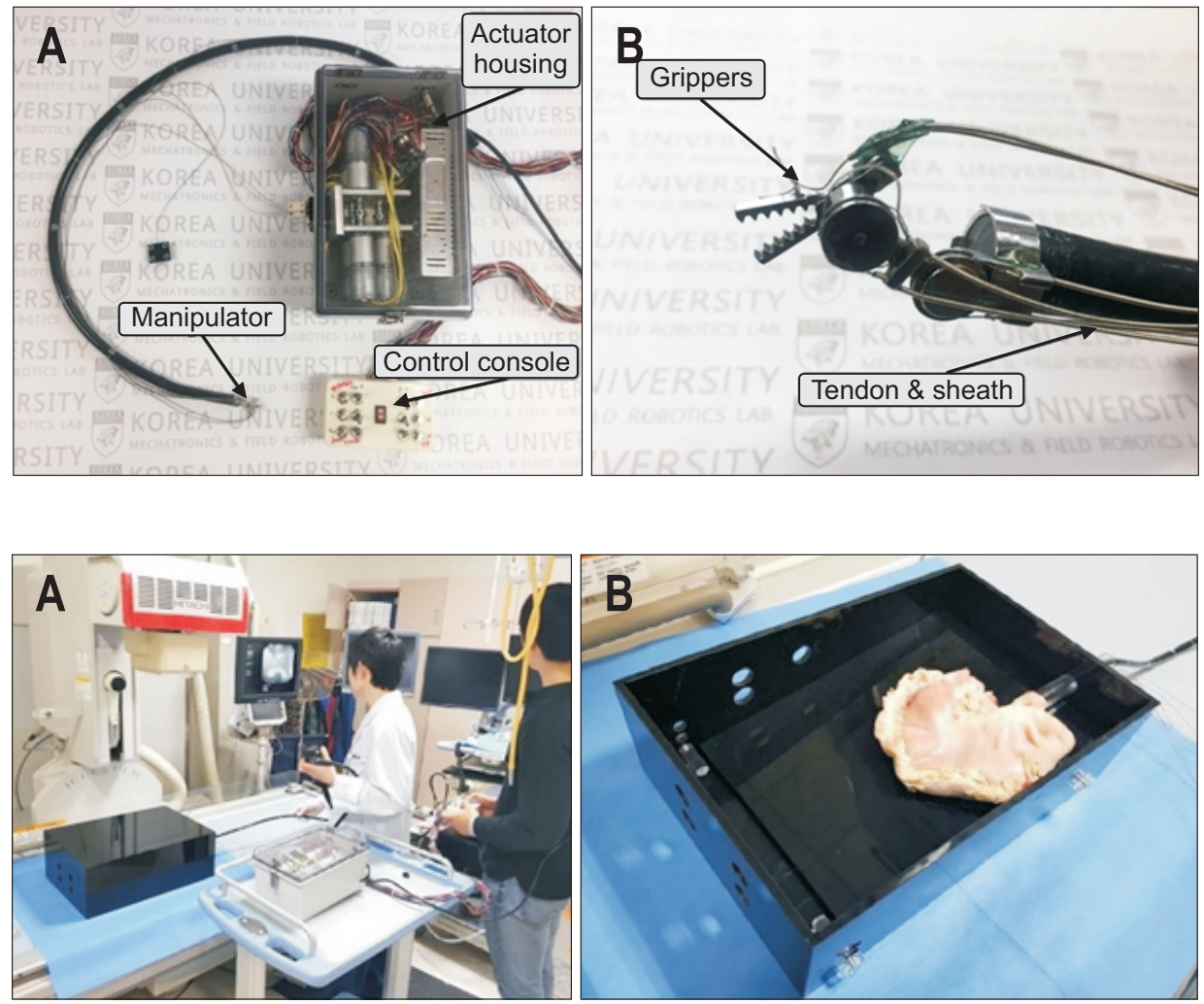

Fig. 2. (A) Overview of the prototype revolute joint-based auxiliary transluminal endoscopic robot (REXTER) system. (B) Minimal manipulator of REXTER.
Fig. 3. (A) Overview of the experimental environment. (B) The in vitro test bed. distal end of a general-use endoscope (GIF-2T240; Olympus) when needed and uninstalled after use (Fig. 2B). This feature makes REXTER convenient to apply in a clinical environment and distinguishes it from other endoscopic surgical robots. The robotic arm has two links (15 mm each) considering the size of the mucosal layer for dissection and has 4 DOFs to enable complex movements required for surgery. The operation of REXTER is based on a tendon-sheath mechanism (TSM), which is an appropriate system in a medical robot. ${ }^{23}$ The TSM is a power transmission system that controls the joints of a robot with a cable when the actuator and robot joint are separated. ${ }^{24}$ The actuator housing is equipped with a motor, controller, and power supply unit. The interface console is used by the surgical assistant to operate REXTER.

\section{Participant classification}

Participants were divided into the following groups $(n=3$, each) to understand the specific effects of the robot-assisted method: skilled group (skilled operators with at least 5 years and 500 cases of experience in ESD) and unskilled group (unskilled operators with less than 3 months and 10 cases of experience in ESD). Each group performed ESD 10 times using each surgical method (conventional and robot-assisted methods). Consequently, in vitro tests were conducted 40 times. One assistant helped with the operation of REXTER. The experimental environment is shown in Fig. 3A.

\section{Sample preparation}

A total of 40 pig stomachs were used in this study. The pigs were bred in a Korean farm for 6 months and weighed approximately $110 \mathrm{~kg}$ each. All stomachs were immediately extracted after killing the pigs on the date of the experiment. The stomachs were refrigerated for 3 hours until the start of the experiment, and remnant food items in the stomachs were removed before the experiment.

\section{In vitro test bed}

In this study, a specifically designed in vitro test bed was used to replicate the endoscope-based surgical environment. An external case was created using nontransparent black acryl that blocks light to replicate an intra-abdominal darkroom environment. Within the case, the slope of the stomach could be adjusted to three levels. The stomach was placed on a panel inside the case, and the esophageal region was connected to a tube that was fixed in the case (Fig. 3B).

\section{RESULTS}

With the conventional method, skilled and unskilled operators showed notable differences in terms of surgical safety and efficiency.

In the skilled group, the conventional and robot-assisted methods did not cause perforation. The mean operation time 
Table 1. Results of Skilled Operators

\begin{tabular}{|c|c|c|c|c|c|c|c|c|}
\hline \multirow{2}{*}{ Procedure no. } & \multicolumn{4}{|c|}{ Conventional method } & \multicolumn{4}{|c|}{ Robot-assisted method } \\
\hline & Perforation & $\begin{array}{l}\text { Procedure time } \\
\text { (min) }\end{array}$ & $\begin{array}{l}\text { DDA } \\
(\mathrm{cm})\end{array}$ & $\begin{array}{c}\text { TPUD } \\
(\mathrm{min} / \mathrm{cm})\end{array}$ & Perforation & $\begin{array}{l}\text { Procedure time } \\
\text { (min) }\end{array}$ & $\begin{array}{l}\text { DDA } \\
(\mathrm{cm})\end{array}$ & $\begin{array}{c}\text { TPUD } \\
(\min / \mathrm{cm})\end{array}$ \\
\hline 1 & No & 8.8 & 3.1 & 2.84 & No & 17.1 & 3.0 & 5.70 \\
\hline 2 & No & 7.6 & 3.1 & 2.45 & No & 15.4 & 4.1 & 3.76 \\
\hline 3 & No & 6.5 & 2.6 & 2.50 & No & 15.7 & 2.8 & 5.61 \\
\hline 4 & No & 6.1 & 1.6 & 3.81 & No & 10.9 & 2.1 & 5.19 \\
\hline 5 & No & 10.9 & 3.2 & 3.41 & No & 11.2 & 2.9 & 3.86 \\
\hline Average & & 7.98 & 2.72 & 3.00 & & 14.06 & 2.98 & 4.82 \\
\hline 6 & No & 15.5 & 3.6 & 4.31 & No & 4.6 & 2.1 & 2.19 \\
\hline 7 & No & 17.7 & 4.7 & 3.77 & No & 7.7 & 2.5 & 3.08 \\
\hline 8 & No & 13.3 & 4.9 & 2.71 & No & 7.7 & 5.0 & 1.54 \\
\hline 9 & No & 18.7 & 3.8 & 4.92 & No & 9.8 & 5.6 & 1.75 \\
\hline 10 & No & 7.7 & 3.2 & 2.41 & No & 12.2 & 5.5 & 2.22 \\
\hline Average & & 14.58 & 4.04 & 3.62 & & 8.4 & 4.14 & 2.16 \\
\hline Overall average & & 11.28 & 3.38 & 3.31 & & 11.23 & 3.56 & 3.49 \\
\hline
\end{tabular}

DDA, diameter of the dissected area; TPUD, time per unit diameter.

Table 2. Results of Unskilled Operators

\begin{tabular}{|c|c|c|c|c|c|c|c|c|}
\hline \multirow{2}{*}{ Procedure no. } & \multicolumn{4}{|c|}{ Conventional method } & \multicolumn{4}{|c|}{ Robot-assisted method } \\
\hline & Perforation & $\begin{array}{l}\text { Procedure time } \\
\text { (min) }\end{array}$ & $\begin{array}{l}\text { DDA } \\
(\mathrm{cm})\end{array}$ & $\begin{array}{c}\text { TPUD } \\
(\mathrm{min} / \mathrm{cm})\end{array}$ & Perforation & $\begin{array}{l}\text { Procedure time } \\
\text { (min) }\end{array}$ & $\begin{array}{l}\text { DDA } \\
(\mathrm{cm})\end{array}$ & $\begin{array}{c}\text { TPUD } \\
(\mathrm{min} / \mathrm{cm})\end{array}$ \\
\hline 1 & No & 29.0 & 2.9 & 10.00 & No & 46.6 & 4.0 & 11.7 \\
\hline 2 & Yes & 13.4 & Piecemeal & NA & No & 27.6 & 2.9 & 9.52 \\
\hline 3 & Yes & 19.0 & 3.0 & 6.33 & Yes & 36.6 & 2.8 & 13.07 \\
\hline 4 & No & 29.9 & 2.3 & 13.00 & No & 40.2 & 5.3 & 7.58 \\
\hline 5 & Yes & 29.7 & 3.2 & 9.28 & No & 32.4 & 3.2 & 10.13 \\
\hline Average & & 24.2 & 2.85 & 9.65 & & 36.68 & 3.64 & 10.4 \\
\hline 6 & No & 31.6 & 4.2 & 7.52 & No & 26.6 & 3.8 & 7.00 \\
\hline 7 & Yes & 19.1 & 2.5 & 7.64 & No & 12.3 & 4.1 & 3.00 \\
\hline 8 & Yes & 16.0 & Piecemeal & NA & No & 22.7 & 4.4 & 5.16 \\
\hline 9 & No & 51.4 & 5.1 & 10.08 & No & 15.1 & 4.7 & 3.21 \\
\hline 10 & Yes & 27.5 & 4.9 & 5.61 & No & 13.8 & 4.1 & 3.37 \\
\hline Average & & 29.12 & 4.18 & 7.71 & & 18.1 & 4.22 & 4.15 \\
\hline Overall average & & 26.66 & 3.52 & 8.68 & & 27.39 & 3.93 & 7.28 \\
\hline
\end{tabular}

DDA, diameter of the dissected area; TPUD, time per unit diameter; NA, not available.

was not significantly different between the conventional and robot-assisted methods $(11.3 \pm 4.8$ and $11.2 \pm 4.0$ minutes, respectively). However, with the robot-assisted method, the operation time decreased as the experiment proceeded (Table 1).

In the unskilled group, perforations occurred in six of 10 tests with the conventional method and in one of 10 tests with the robot-assisted method. Thus, surgical safety improved in unskilled operators with robotic assistance. Similar to the findings in the skilled group, the mean operation time was not significantly different between the conventional and robot-assisted methods (26.7 \pm 10.9 and $27.4 \pm 10.7$ minutes, respectively). However, there was an approximately $16 \%$ reduction in the time per unit diameter with the robot-assisted method (Table 2).

During the collection of dissected tissue, piecemeal dissection was performed in two cases by using the conventional method, and en bloc dissection was performed in all cases by using the robot-assisted method. In both skilled and unskilled operators with the robot-assisted method, the operation time decreased as the experiment proceeded (Tables 1 and 2). 


\section{DISCUSSION}

We developed a robot-assisted system as a surgical instrument to overcome the limitations of an endoscope. With regard to the target surgical method ESD, the surgical results for skilled and unskilled operators were preliminarily identified, and pilot tests were conducted to examine the benefits of robotic assistance in both types of operators. Comparative assessment of the conventional method showed that skilled and unskilled operators had large differences in terms of surgical safety and efficiency. This difference might explain the current extensive research on the development of endoscopic assistive devices for unskilled operators. Here, the surgical safety of unskilled operators improved with robotic assistance.

The perforation incidence during ESD is an important factor for determining surgical safety. ${ }^{25,26}$ In this study, the perforation incidence was recorded in all tests, and the causes were analyzed. Skilled operators did not cause perforation in any tests, whereas unskilled operators caused perforation in six of 10 tests with the conventional method. The major causes of perforation were as follows. The first cause was the inability to clearly visualize the area to be dissected. During dissection of the submucosal layer, the part of the membrane that had already been dissected covered the area that had to be subsequently dissected. Therefore, the operators could not visualize the area to be dissected and had to perform dissection according to their intuition. Skilled operators could complete the dissection even without clear visualization because of their experience. Unskilled operators lacked surgical experience; thus, they were more likely to make mistakes in this situation, consistent with the high perforation incidence in this experiment. The second cause was the difference in controlling the electrical knife when it approached the gastric wall. Skilled operators performed the task with the knife parallel to the wall as much as possible. Eventually, using these electric knives effectively prevents perforation in many difficult situations. During surgery with the conventional method, unskilled operators attempted to secure area visibility for dissection by placing the lesion upward according to the direction of gravity. This positioning caused the dissected tissue to hang downward, which resolved the issue of the flap covering the dissection area and exposed the dissection area. However, with this approach, the lesion was present at the top of the stomach, and the endoscope was present at the bottom of the stomach. Therefore, the knife was almost perpendicular to the stomach wall, and several perforations occurred (Fig. 4A). Alternatively, unskilled operators could maintain the cutter parallel to the stomach wall with the robot-assisted method, and this placement possibly reduced the perforation incidence (Fig. $4 \mathrm{~B})$.

In this experiment, the total operation time did not significantly differ depending on the surgical method. In general, the stages of ESD include marking, injection, precutting, and main cutting. Although the robot-assisted method included an additional step of lifting the mucous membrane, the other tasks were easier compared to those with the use of the conventional method. This ease of use might have offset the time taken for the extra step; therefore, the total operation time was not significantly different between the two methods. This experiment was conducted without a separate preliminary training session; thus, the operators were not familiar with robotic assistance. However, once the experiment proceeded, the operators quickly adjusted to the robot-assisted method and showed a decreased operation time in the later stage of the experiment compared with that in the earlier stage. These findings suggest that the use of robots can make ESD easier.

This study has some limitations. The experiment was conducted using the second REXTER prototype, which can perform all functions of REXTER, but its durability is not as reliable. Therefore, we could not conduct many experiments to obtain statistically significant values, and only the tendency of the effect on the surgery was examined through 40 pilot tests. Precise precutting is another important factor in the evaluation of ESD procedure. In this study, the precutting step was performed using only the endoscope and electronic dual knife without the robot assistant. Therefore, the precision of precutting was not evaluated in this study. However, this experiment proved worthy of further experimentation with more precisely engineered robots. A third prototype for clinical trials is under development.

Several improvements should be made before conducting survival in vivo tests and clinical trials using REXTER. First, the robotic arm should be further miniaturized. The overtube provides esophageal protection such that the robot is able to
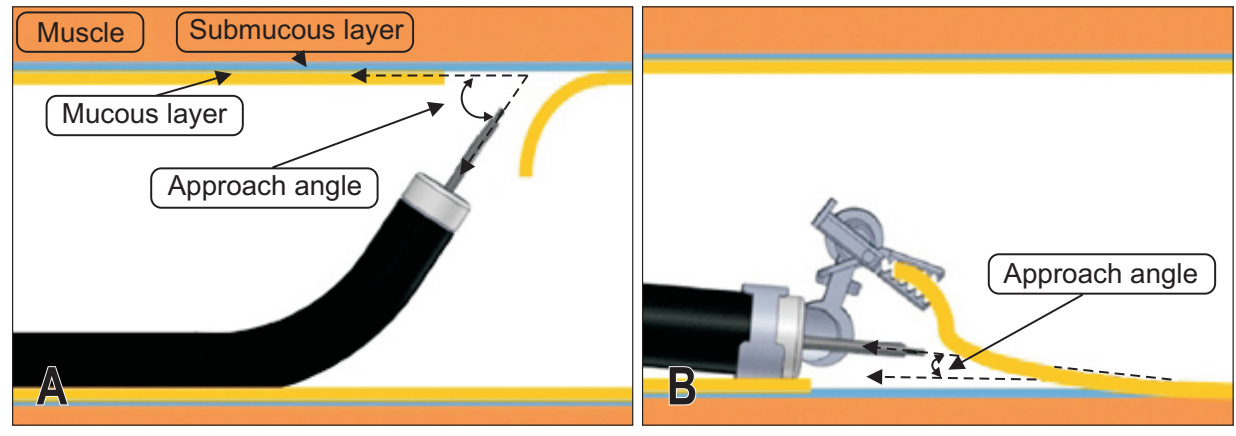

Fig. 4. (A) Configuration of the stomach wall and endoscope with the conventional approach. (B) Configuration of the stomach wall and endoscope with revolute joint-based auxiliary transluminal endoscopic robot (REXTER). 
pass through the esophagus and enter the stomach without damaging the esophagus. REXTER should be sized to easily pass through the overtube. In the present study, a two-channel endoscope (GIF-2T240 endoscope; Olympus, diameter, $11.8 \mathrm{~mm}$ ) was used, and the maximum diameter of its distal end was 17 $\mathrm{mm}$ when the robotic arm was attached. Although REXTER is smaller than most other endoscopic robots, the size should be further reduced for clinical trials. At present, a robot suitable for a smaller diameter endoscope (GIF-Q260; Olympus, diameter, $9.2 \mathrm{~mm}$ ) is under development. Second, the current interface used for REXTER is not intuitive enough for doctors or nurses to operate. In this study, an engineer who helped develop the REXTER system participated in the experiments as the surgical assistant and operated the robot. For clinical trials, a more intuitive, ergonomic interface should be designed to provide easy use of the robot. Third, materials that are considered harmless to the human body should be used. The current test robot was developed using SUS304 stainless steel for in vitro testing. Other materials for in vivo testing are under development.

In conclusion, REXTER showed greater improvements in surgical safety among unskilled operators than among skilled operators. Thus, our assistive robotic arm was beneficial for ESD. Our findings suggest that endoscopic assistive robots may have positive effects on surgical safety. Although no remarkable changes in operation time were observed, future studies will likely help reduce the operation time. These future studies should also attempt to reduce the size of endoscopic surgical robots and further advance the TSM for application in clinical practice. In addition, an ergonomic, intuitive interface should be developed to ensure surgical safety as well as efficiency among skilled and unskilled operators.

\section{CONFLICTS OF INTEREST}

No potential conflict of interest relevant to this article was reported.

\section{ACKNOWLEDGEMENTS}

This work was supported by the National Research Foundation of Korea (NRF) grant funded by the Korea government (MSIP) (No. 2017R1C1B2006425). This research was supported by a grant of the Korea Health Technology R\&D Project through the Korea Health Industry Development Institute (KHIDI), funded by the Ministry of Health \& Welfare, Republic of Korea (grant number: HI14C3477).

\section{AUTHOR CONTRIBUTIONS}

B.G.K. designed and manufactured the robot system and wrote the manuscript; H.S.C. designed the study and in-vitro experimentation, analyzed the result and reviewed the manu-

script; S.H.P. and J.H.H. assisted robot design and manufacturing; J.M.L. and S.H.K. assisted in-vitro study and performed ESD as a skilled operator; H.J.C. supervised the porcine model study and experiments; B.K. reviewed the medical contents in the manuscript and supervised experiments; D.H. reviewed the manuscript and supervised development of the robot system.

\section{ORCID}

Byung Gon Kim

Daehie Hong

Bora Keum

\section{REFERENCES}

1. Dogangil G, Davies BL, Rodriguez y Baena F. A review of medical robotics for minimally invasive soft tissue surgery. Proc Inst Mech Eng H 2010;224:653-679.

2. Pearl JP, Ponsky JL. Natural orifice translumenal endoscopic surgery: a critical review. J Gastrointest Surg 2008;12:1293-1300.

3. Kalloo AN, Singh VK, Jagannath SB, et al. Flexible transgastric peritoneoscopy: a novel approach to diagnostic and therapeutic interventions in the peritoneal cavity. Gastrointest Endosc 2004;60:114-117.

4. Abbott DJ, Becke C, Rothstein RI, Peine WJ. Design of an endoluminal NOTES robotics system. Proceedings of the International Conference on Intelligent Robots and Systems; 2007 Oct 29-Nov 2; San Diego, CA, USA.

5. Ono H, Kondo H, Gotoda T, et al. Endoscopic mucosal resection for treatment of early gastric cancer. Gut 2001;48:225-229.

6. Hirao M, Masuda K, Asanuma T, et al. Endoscopic resection of early gastric cancer and other tumors with local injection of hypertonic saline-epinephrine. Gastrointest Endosc 1988;34:264269.

7. Kato M, Gromski M, Jung Y, Chuttani R, Matthes K. The learning curve for endoscopic submucosal dissection in an established experimental setting. Surg Endosc 2013;27:154-161.

8. Ma MX, Bourke MJ. Endoscopic submucosal dissection in the West: current status and future directions. Dig Endosc 2018;30:310-320.

9. Yeung BP, Gourlay T. A technical review of flexible endoscopic multitasking platforms. Int J Surg 2012;10:345-354.

10. Choi HS, Chun HJ. Accessory devices frequently used for endoscopic submucosal dissection. Clin Endosc 2017;50:224-233.

11. Song Y, Choi HS, Kim K, et al. A simple novel endoscopic successive suture device: a validation study for closure strength and reproducibility. Endoscopy 2013;45:655-660.

12. Choi HS, Chun HJ. Recent trends in endoscopic bariatric therapies. Clin Endosc 2017;50:11-16.

13. Joe S, Lee D, Park J0, Park S, Kim B. Input signal effects on the locomotion of a robotic colonoscope activated by a flexible shaft. Int J Precis Eng Manuf 2017;18:461-465. 
14. Kim Y, Choi HS, Kim K, et al. A novel endoscopic surgical device for gastric volume reduction bariatric surgery. Int J Precis Eng Manuf 2017;17:467-472.

15. Isshi K, Tajiri H, Fujisaki J, et al. The effectiveness of a new multibending scope for endoscopic mucosal resection. Endoscopy 2004;36:294-297.

16. Sumiyama K, Kaise M, Nakayoshi T, et al. Combined use of a magnifying endoscope with a narrow band imaging system and a multibending endoscope for en bloc EMR of early stage gastric cancer. Gastrointest Endosc 2004;60:79-84.

17. Spaun G0, Zheng B, Swanström LL. A multitasking platform for natural orifice translumenal endoscopic surgery (NOTES): a benchtop comparison of a new device for flexible endoscopic surgery and a standard dual-channel endoscope. Surg Endosc 2009;23:2720-2727.

18. Ikeda K, Sumiyama K, Tajiri H, Yasuda K, Kitano S. Evaluation of a new multitasking platform for endoscopic full-thickness resection. Gastrointest Endosc 2011;73:117-122.

19. Phee SJ, Ho KY, Lomanto D, et al. Natural orifice transgastric endoscopic wedge hepatic resection in an experimental model using an intuitively controlled master and slave transluminal endoscopic robot (MASTER). Surg Endosc 2010;24:2293-2298.

20. Phee SJ, Low SC, Huynh VA, Kencana AP, Sun ZL, Yang K. Mas- ter and slave transluminal endoscopic robot (MASTER) for natural orifice transluminal endoscopic surgery (NOTES). Conf Proc IEEE Eng Med Biol Soc 2009;2009:1192-1195.

21. Thompson CC, Ryou M, Soper NJ, Hungess ES, Rothstein RI, Swanstrom LL. Evaluation of a manually driven, multitasking platform for complex endoluminal and natural orifice transluminal endoscopic surgery applications (with video). Gastrointest Endosc 2009;70:121-125.

22. Chiu PW, Phee SJ, Bhandari P, et al. Enhancing proficiency in performing endoscopic submucosal dissection (ESD) by using a prototype robotic endoscope. Endosc Int Open 2015;3:E439-E442.

23. Le HM, Do TN, Phee SJ. A survey on actuators-driven surgical robots. Sens Actuators A Phys 2016; 247:323-354.

24. Phee SJ, Low SC, Dario P, Menciassi A. Tendon sheath analysis for estimation of distal end force and elongation for sensorless distal end. Robotica 2010;28:1073-1082.

25. Oda I, Saito D, Tada M, et al. A multicenter retrospective study of endoscopic resection for early gastric cancer. Gastric Cancer 2006;9:262-270.

26. Sugimoto T, Okamoto M, Mitsuno Y, et al. Endoscopic submucosal dissection is an effective and safe therapy for early gastric neoplasms: a multicenter feasible study. J Clin Gastroenterol 2012;46:124-129. 